\title{
Electropolymerization of Aniline on Plastically Deformed Pd Surface: Structure at Micro- and Nano-Scale
}

\author{
Ali Eftekhari, ${ }^{\dagger}$ Mahmood KaZemzad, and Mansoor KeyAnPour-Rad \\ Laboratory of Electrochemistry, Materials and Energy Research Center, P. O. Box 14155-4777, Tehran, Iran
}

(Received October 14, 2005; Accepted November 14, 2005; Published April 15, 2006)

\begin{abstract}
Electrochemical polymerization of aniline on the surface of a plastically deformed Pd electrode was investigated. Initially, the Pd electrode was subject of plastic deformation as a result of hydrogen insertion/extraction. Plastically deformed structure of the substrate has a significant influence on the electropolymerization process. In this case, an adherent film with smooth surface is formed. Although the plastically deformed surface is rougher than the conventional Pd surface, the polyaniline film electropolymerized is smoother in both micro- and nano-scale. This is of particular interest for applied purposes, as it guarantees higher stability of the conductive polymer. In addition, these results indicate the importance of surface structure of substrate electrode on electropolymerization process. On the other hand, it suggests Pd as a suitable substrate for the preparation of thin films of conductive polymers.
\end{abstract}

[DOI 10.1295/polymj.38.329]

KEY WORDS Polyaniline / Conductive Polymers / Plastic Deformation / Pd Substrate / Electropolymerization / Film Morphology / Nanostructure /

Substrate electrodes play important roles in electrochemical polymerization processes to form conductive polymers films. Electropolymerization on various substrate electrodes have been widely investigated in the literature. ${ }^{1-9}$ In this context, chemical activity of substrate electrode may provide new opportunities for the electropolymerization processes. It has been described that oxidizable metals (in respect to the electrolyte medium, i.e. a suitable electrolyte with ability to oxidize the specified metallic electrode) will be chemically oxidized before the deposition of conductive polymer. ${ }^{9}$ This phenomenon leads to the formation of a metal oxide film, which provides more suitable sites for the deposition of conductive polymer. In other words, the porous structure of this metal oxide layer allows penetration of the electroactive film slightly within the substrate surface..$^{10}$ This is accompanied by stronger stability of the conductive polymer attached to the substrate surface. In addition, rough structure of the metal oxide layer assists nucleation and growth of the conductive polymer.

Although effect of various metallic substrates on electropolymerization processes has been extensively studied, less attention has been paid to the influence of surface structure of a specified substrate surface. Plastic deformation is a well-known phenomenon in solid-state science, which results in severe surface structural changes. ${ }^{11-13}$ In the present work, we wish to use a Pd electrode, which was subject of plastic deformation, as a new substrate surface for the deposition of conductive polymers.

\section{EXPERIMENTAL}

Effect of plastic deformation, as a result of electrochemical insertion/extraction of hydrogen into/from Pt electrode, on its surface structure has been previously reported. ${ }^{11-13}$ Only surface structure of a plastically deformed Pd electrode may affect electropolymerization process due to electron transfer across the electrode/electrolyte interface, and plastic deformation in bulk, which is of particular interest in solid-state physics, has no influence on the interfacial deposition of conductive polymer. Therefore, the previous surface studies ${ }^{11-13}$ satisfy the requirement of the present work to use a typical plastically deformed Pd with known surface structure. The plastic deformation was induced to the Pd electrode in accordance with the procedure followed in the previous works. ${ }^{11-13}$ Briefly, hydrogen was injected into the Pd bulk from an aqueous solution of $0.1 \mathrm{M} \mathrm{NaOH}$ by applying a constant current of $13.5 \mathrm{~mA} / \mathrm{cm}^{2}$ for a certain time. According to statistical comparison of Pd electrodes with various degrees of plastic deformation, ${ }^{12,13}$ this galvanostatic hydrogen injection at a short time of $c a$. $900 \mathrm{~s}$ results in a slight plastic deformation. As will be reported below, the influence of plastically deformed substrate on electropolymerization process is very significant, thus, a slight plastic deformation can represent the phenomenon. On the other hand, surface roughnesses of two Pd electrodes with and without plastic deformation are approximately the same.

${ }^{\dagger}$ To whom correspondence should be addressed (Tel: +98-261-621-0009, Fax: +98-261-620-1888, E-mail: eftekhari@merc.ac.ir). 
(A)

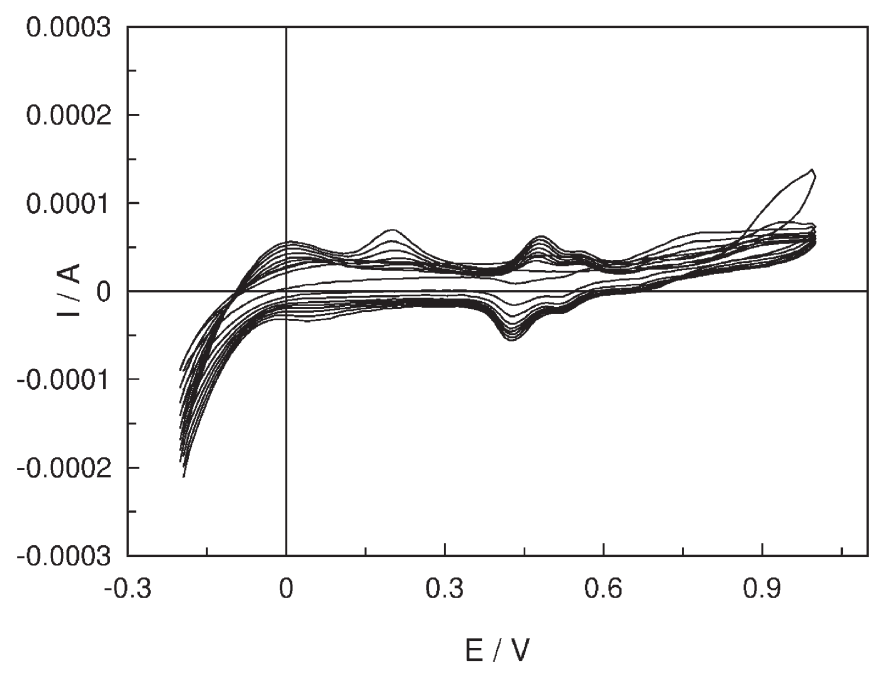

(B)

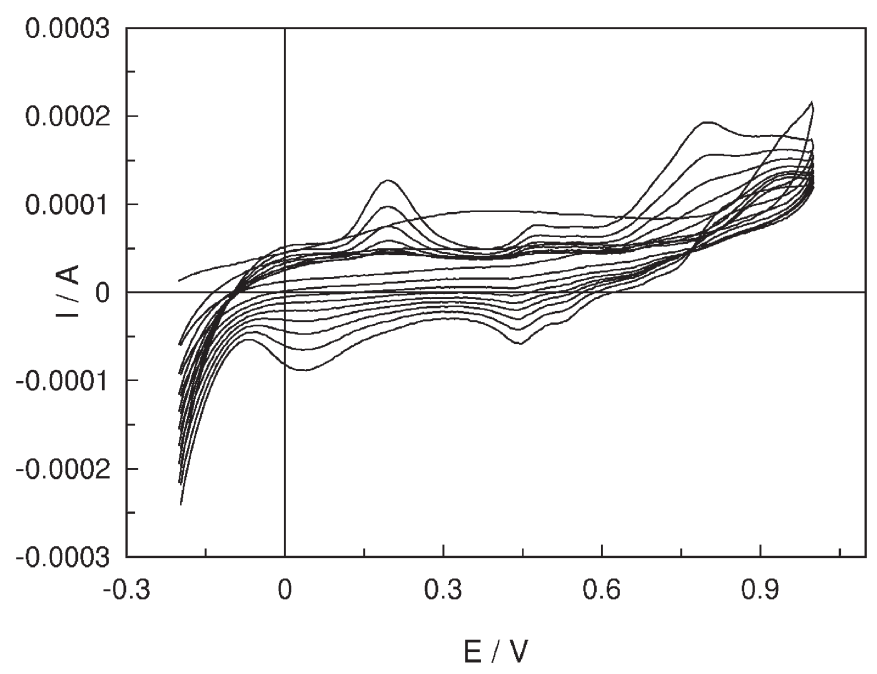

Figure 1. Potentiodynamic growth of the polyaniline film on (A) a fresh Pd electrode and (B) a plastically deformed Pd electrode from a solution containing $30 \mathrm{mM}$ aniline and $0.5 \mathrm{M} \mathrm{H}_{2} \mathrm{SO}_{4}$. Potential cycling was performed with scan rate of $50 \mathrm{mV} / \mathrm{s}$.

Electrochemical polymerization of aniline on the Pd substrate electrode was performed under conventional condition. A thin film of polyaniline was deposited by 9 successive potential cycles between -0.2 and $1.0 \mathrm{~V}(v s . \mathrm{Ag} / \mathrm{AgCl})$ with a scan rate of $50 \mathrm{mV} /$ s. The electrolyte solution was $30 \mathrm{mM}$ aniline in $0.5 \mathrm{M} \mathrm{H}_{2} \mathrm{SO}_{4}$ as supporting electrolyte. Electrochemical experiments were performed using a Metrohm 746VA potentiostat with its original three-electrode cell. Scanning electron microscopic images were recorded using a Cambridge electron microscope model Steroscan 360. Small-angle X-ray scattering (SAXS) investigations were performed using a Phillips PW 3710 diffractometer employing a rotating X-ray generator under appropriate condition.

\section{RESULTS AND DISCUSSION}

Although palladium is a suitable metallic substrate for the deposition of conductive polymers as well as other noble metals, less attention has been paid to Pd for this purpose. Instead, Pd is a common metallic additive to be incorporated into polymer matrix. ${ }^{14-21}$ This leads to superior catalytic activity due to significant versatility of palladium for $\mathrm{C}-\mathrm{C}$ bond formations. ${ }^{22}$ It is believed that this feature is also useful when Pd is used as substrate for a conductive polymer (at least for thin films). However, it is just restricted to few reports regarding chemical polymerization on palladium surface, ${ }^{23,24}$ where the substrate has no critical role on the polymerization process. However, electropolymerization, which is accompanied by complicated interfacial processes, is also efficiently succeeded on $\mathrm{Pd}$ substrate surface (Figure 1A). This typical electrochemical synthesis of aniline displays characteristic peaks of polyaniline growth.

Figure 1 compares potentiodynamic electropolymerization of aniline onto fresh and plastically de- 
(A)

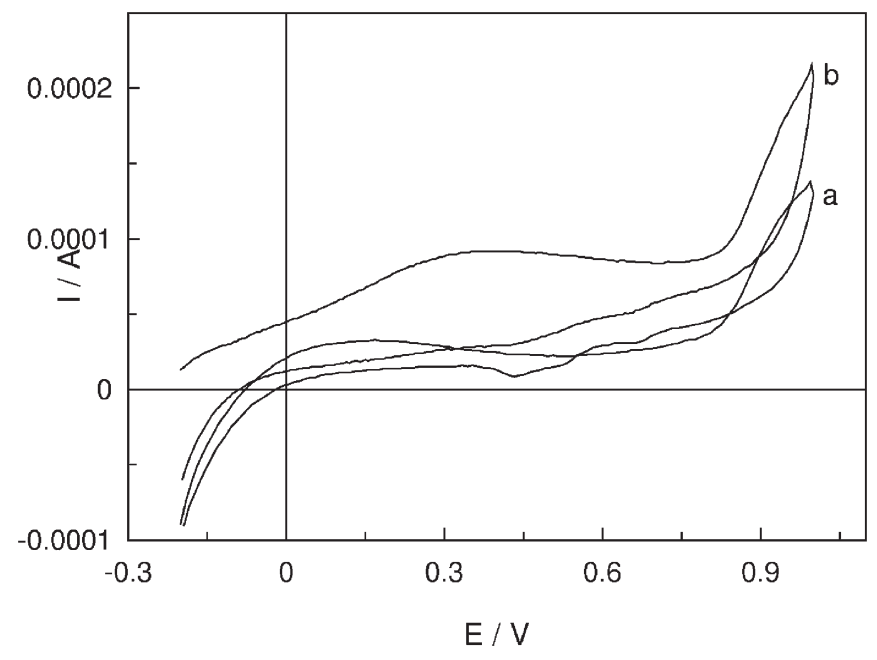

(B)

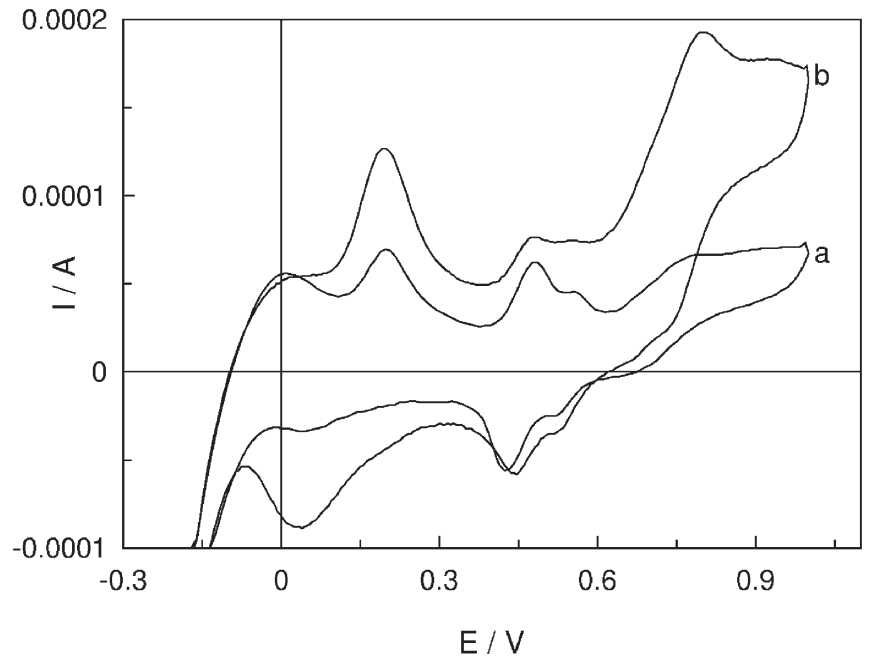

Figure 2. Cyclic voltammetric behavior of the electropolymerization processes corresponding to (A) the first and (B) 9 th cycles. The symbols $a$ and $b$ refer to the fresh and plastically deformed Pd electrodes, respectively.

formed Pd substrate electrodes. Although both electrochemical syntheses display characteristic peaks of polyaniline growth, the charge passed and subsequently the amount of conductive polymer generated is significantly higher for the case of plastically deformed Pd substrate. This is not due to the specific area of the substrate surface since it has been normalized, and increase of this difference (between two cases) is more significant for higher cycle numbers. It is indeed indicative of the fact that electropolymerization process is more successful on plastically deformed Pd substrate. This is of particular interest for applied purposes due to advantageous efficiency of the polymerization process.

Another peculiar behavior of the plastically deformed $\mathrm{Pd}$ electrode for the electropolymerization process is unusual cyclic voltammograms recorded at the first scan (Figure 2A). As it has been discussed in ref 9 , a necessary requirement for the occurrence of electropolymerization is initial oxidation of substrate surface. This process needs a certain amount of charge depending on the type of substrate material and the electrolyte solution. It is obvious that a higher amount of charge is required for the oxidation of plastically deformed Pd electrode, which can be attributed to higher electrocatalytic activity of this complicated structure. This issue is also confirmed by the strategy followed in ref 9, by means of galvanostatic investigations, though we disregard discussion of such results here to keep the phenomenological aim of the present report. The cyclic voltammograms (the first scan) of two different cases reveal that electropolymerization slightly occurs on the fresh Pd electrode even at the first scan (Figure 2A). Whereas, the cyclic voltammetric behavior of the plastically deformed Pd electrode displays no characteristic peaks of electropolymerization (Figure 2A). It is believed that this severe oxidation process in the course of first scan 

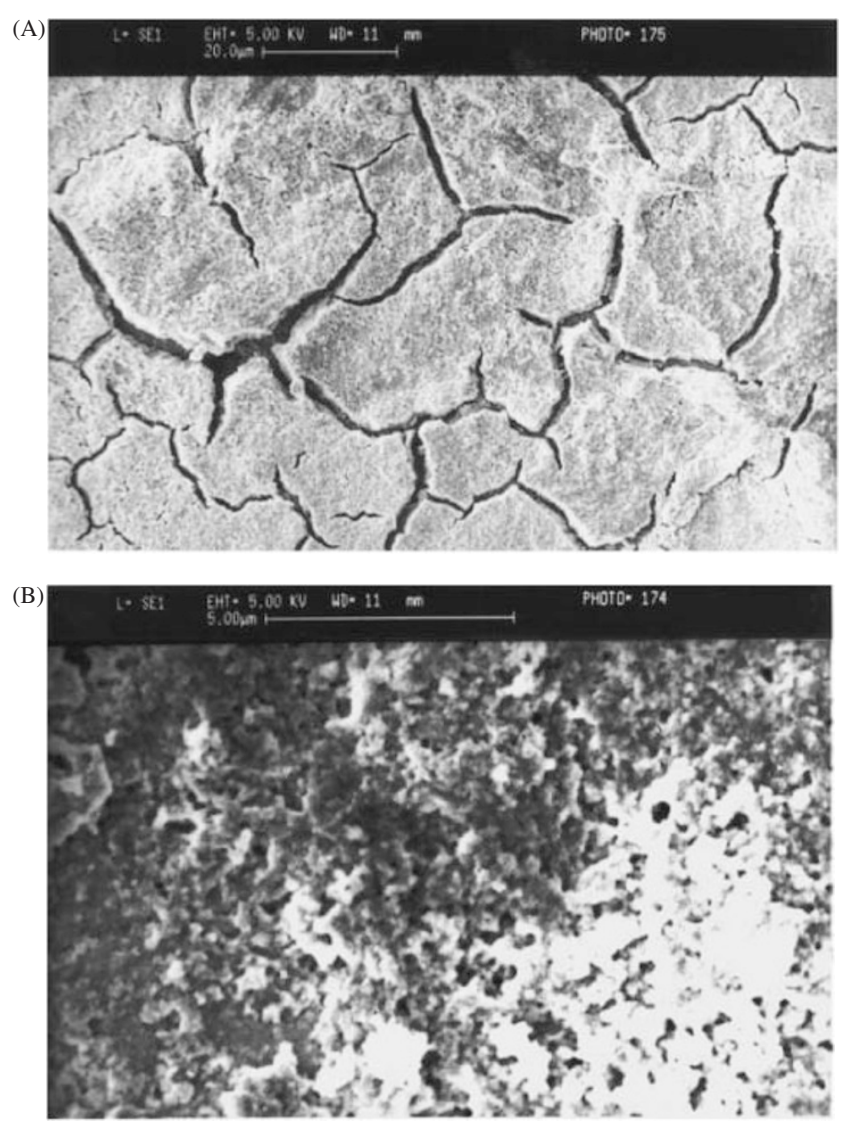

Figure 3. Typical SEM images of the polyaniline film electrodeposited onto the fresh Pd substrate electrode. The scale bars are 20 and $5 \mu \mathrm{m}$ for (A) and (B), respectively.

leads to the formation of a suitable surface for subsequent growth of polyaniline. Similarly, this issue has been discussed for the case of electropolymerization of aniline onto iron substrate electrode. ${ }^{9}$

Comparison of cyclic voltammograms illustrated in Figure 2A also suggests higher electrocatalytic activity of the plastically deformed Pd electrode rather than the fresh Pd electrode. This is not only related to the first cycle, when the metallic electrodes are approximately bare, but also for polyaniline-coated electrodes (Figure 2B). This indicates that the internal contact of the polymer first layer with the substrate surface at the electrode/electrolyte interface controls subsequent growth of the conductive polymer. In other words, plastically deformed structure of the Pd substrate acts as active centers for polymer growth. The thin polymer film formed on the Pd substrate surface has a strong electrocatalytic activity.

In analyzing the results obtained from electrochemical investigations, we briefly noted the peculiar polymer growth on the plastically deformed Pd surface. However, we leave this issue for an alternative work devoted to mechanism of polymerization and concentrate on a more informative effect of this peculiar substrate surface, i.e. surface morphology. The influence
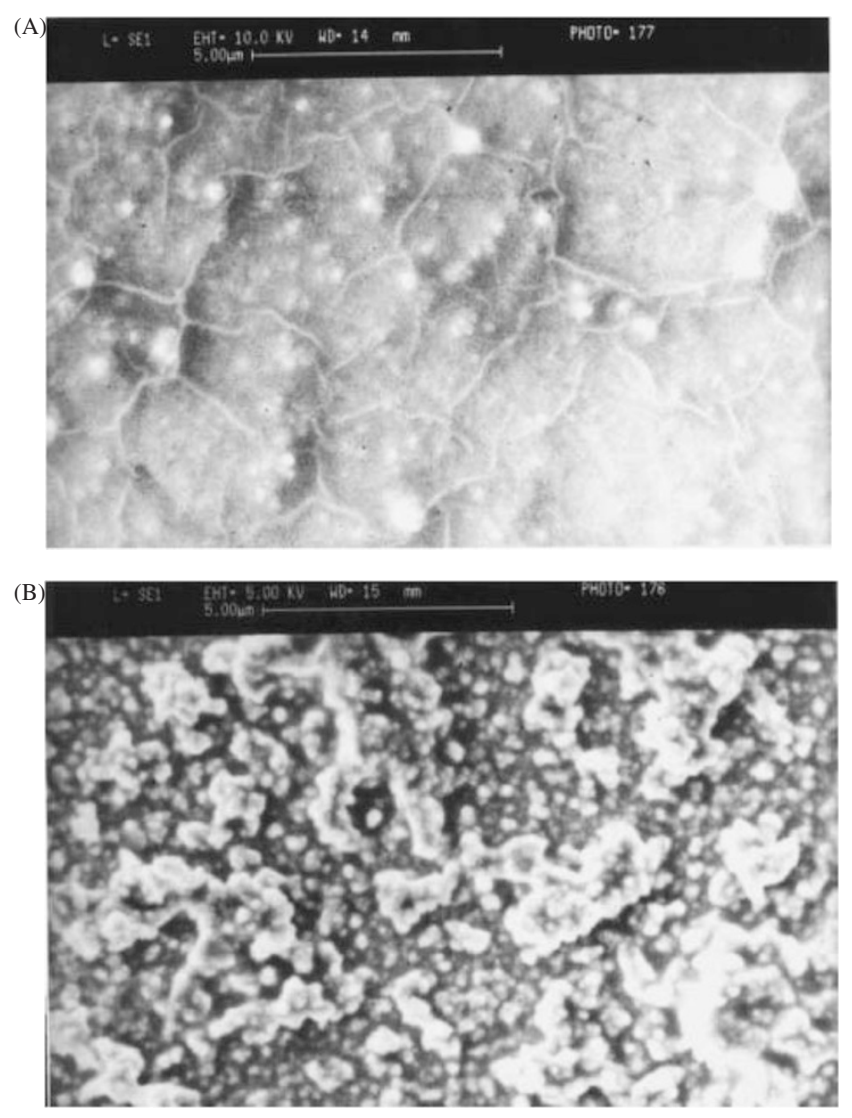

Figure 4. Typical SEM images of the polyaniline film electrodeposited onto the plastically deformed Pd substrate electrode. The scale bars for both images are $5 \mu \mathrm{m}$.

of plastic deformation of Pd on its surface morphology in both micro- and nano-scale has been extensively studied by means of fractal geometry. ${ }^{12,13}$

Figure 3A shows a typical SEM image of the polyaniline film electrodeposited onto a fresh Pd substrate electrode. It is obvious that the film does not have sufficient internal adherent. Thus, huge cracks injure continuity of the polyaniline film. It is evident that this is unfavorable, since results in low stability of the polymer film and fast breakdown. Although the appearance of such cracks in the film microstructure confirms incontinuty, an excellent homogeneity is observable in nanostructure of the polyaniline film (Figure 3B). This nanostructure of the conductive polymer provides an opportunity for better diffusion of counter ion through the polymer matrix and consequently better ionic conductivity. Disregarding the microstructure of the polyaniline film (Figure 3A), its nanostructure is similar to the polyaniline film electrodeposited onto conventional Pt substrate electrode ( $c f$. ref 25). In fact, the main disadvantage of electropolymerization on Pd electrode is the appearance of such huge cracks.

The film morphology is significantly different for the polyaniline film electrodeposited onto the plastically deformed Pd substrate surface (Figure 4A). The 
size of the above mentioned cracks is significantly lower than those formed within the polyaniline film deposited on fresh Pd electrode. On the other hand, it seems that walls of such cracks tend to be welded (Figure 4A). In fact, electropolymerization on plastically deformed Pd electrode results in the formation of a continuous and dense film. Figure 4B shows the film morphology where the cracks disappear. This indicates that the continuity observed for the polyaniline film electrodeposited on plastically deformed Pd electrode is not due to welding of cracks. In other words, polymer growth on Pd surface (when the electropolymerization starts from a plastically deformed interface) tends to form continuous film attached to the substrate surface.

Since the plastic deformation induced to the $\mathrm{Pd}$ electrode has a significant nanostructure feature, ${ }^{13}$ it is necessary to inspect the film morphology in nanoscale. Both plastic deformation induced to the substrate surface and electropolymerization on complicated structure of a plastically deformed surface are not homogeneous processes resulting in the formation of monotonous surface. Due to such local differences in the surface structure, conventional scanning probe (SPM) techniques are not appropriate from statistical point of view. Thus, we use an efficient technique to obtain a statistical data owing to all regions of the surfaces. Small-angle X-ray scattering (SAXS) is one of the most reliable techniques for surface analysis in the scale of $0.5-200 \mathrm{~nm}^{26}$

A simple comparison of SAXS patterns of the polyaniline films electrodeposited onto two different substrate electrodes (Figure 5) reveals that the normalized SAXS intensity is smaller for the case of plastically deformed Pd substrate. This indicates a smoother surface in the scale under investigation, as

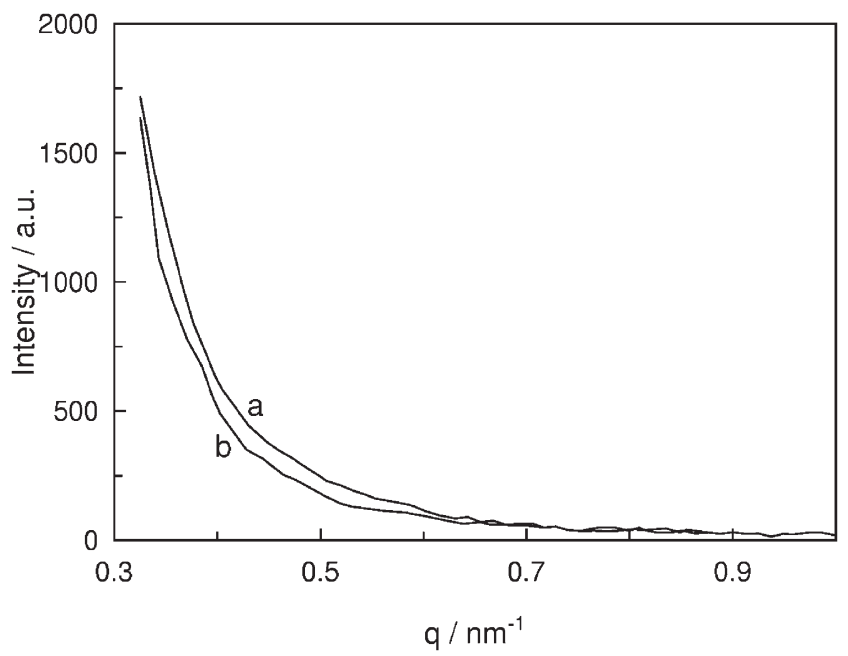

Figure 5. SAXS patterns of the polyaniline films electrodeposited onto the fresh (a) and plastically deformed (b) Pd surfaces. the curve slope is smaller. This is a peculiar behavior, since the roughness of the plastically deformed Pd surface was significantly higher than that of the fresh Pd surface ( $c f$. the statistical comparison of two cases in ref 13). This provides a strong evidence for the fact that electropolymerization on plastically deformed substrate leads to the formation of smooth (or in a sense, ordered) polymer film.

Of course, the results obtained from SAXS investigations can be used to compare two different cases from geometrical point of view; however, this simple judgment satisfies the requirement of the present study. The fractal dimension can be simply estimated by plotting the SAXS patterns in a logarithmic scale in accordance with the method described in ref 13. According to the experimental data, the fractal dimension of the polyaniline film electrodeposited onto the fresh Pd electrode is 2.65 , which is close to the conventional case of $\mathrm{Pt}$ substrate electrode. Whereas, the fractal dimension of the polyaniline film electrodeposited onto the plastically deformed Pd electrode is 2.53. According to the concept of fractal geometry, this tiny difference suggests a significant difference in the surface structures.

In general, the above mentioned phenomenon, i.e. peculiar electropolymerization on plastically deformed substrate, is due to complicated structure generated as a result of plastic deformation, not conventional surface roughness (as checked for a similar roughened Pd electrode). It is worth noting that plastic deformation is also accompanied by the formation of surface defects, and it is known that surface defects significantly affect electrochemical processes. ${ }^{27}$ However, such probable surface defects were healed by simple thermal treatment before electrochemical experiments. In fact, the only possible reason for the occurrence of such phenomenon is special structure appeared on the Pd lattice as a result of plastic deformation. Indeed, this concise report proposes a new opportunity to use plastically deformed substrate for the preparation of thin films for specified applications.

\section{CONCLUSIONS}

Electropolymerization processes, as typically investigated for polyaniline, are strongly dependent on the surface structure of substrate electrode. As well as conventional surface roughness, complicated surface structural changes of the substrate surface may lead to peculiar polymerization process. Plastically deformed Pd electrode was introduced as an excellent substrate surface for electrochemical polymerization. Since complicated structure of a plastically deformed surface can act as suitable centers for polymer growth, the polymer film deposited on the substrate surface is 
denser in comparison with that deposited on conventional substrate. In addition, the lateral film morphology is also highly dependent on the substrate structure. The film electrodeposited onto plastically deformed substrate is continuous and smooth. It is accompanied by better electrochemical efficiency (as lower charge is required for the electropolymerization), and the film deposited has better stability. Therefore, employing plastically deformed substrate surface is an efficient approach for the preparation of thin films of conductive polymers for applied purposes.

\section{REFERENCES}

1. B. Zaid, S. Aeiyach, and P. C. Lacaze, Synth. Met., 65, 27 (1994).

2. I. Marawi, A. Khaskelis, A. Galal, J. F. Rubinson, R. P. Popat, F. J. Boerio, and H. B. Mark, Jr., J. Electroanal. Chem., 434, 61 (1997).

3. S. Aeiyach, E. A. Bazzaoui, and P.-C. Lacaze, J. Electroanal. Chem., 434, 153 (1997).

4. T. Zalewska, A. Lisowska-Oleksiak, S. Biallozor, and V. Jasulaitiene, Electrochim. Acta, 45, 4031 (2000).

5. A. Eftekhari, Synth. Met., 125, 295 (2001).

6. M. Bazzaoui, E. A. Bazzaoui, L. Martins, and J. I. Martins, Synth. Met., 130, 73 (2002).

7. V. Shinde, S. Chaudhari, P. P. Patil, and S. R. Sainkar, Mater. Chem. Phys., 82, 622 (2003).

8. K. G. Conroy and C. B. Breslin, Electrochim. Acta, 48, 721 (2003).

9. A. Eftekhari, Synth. Met., 145, 211 (2004).

10. A. Eftekhari, Solid State Ionics, 167, 237 (2003).
11. J.-N. Han, M. Seo, and S.-I. Pyun, J. Electroanal. Chem., 514, 118 (2001).

12. A. Eftekhari, Physica B, accepted. Preprint is available at http://arxiv.org/physics/0408072.

13. A. Eftekhari, M. Kazemzad, M. Keyanpour-Rad, and M. A. Bahrevar, Appl. Surf. Sci., 242, 82 (2005).

14. N. Cioffi, L. Torsi, I. Losito, L. Sabbatini, P. G. Zambonin, and T. Bleve-Zacheo, Electrochim. Acta, 46, 4205 (2001).

15. M. T. Giacomini, M. Balasubramanian, S. Khalid, J. McBreen, and E. A. Ticianellia, J. Electrochem. Soc., 150, A588 (2003).

16. M. Hasik, A. Bernasik, A. Adamczyk, G. Malata, K. Kowalski, and J. Camra, Eur. Polym. J., 39, 1669 (2003).

17. A. Drelinkiewicz, J. Stejskal, A. Waksmundzka, and J. W. Sobczak, Synth. Met., 140, 233 (2004).

18. J.-E. Park, S.-G. Park, A. Koukitu, O. Katozaki, and N. Oyama, Synth. Met., 140, 121 (2004).

19. M. Hasik, E. Wenda, C. Paluszkiewicz, A. Bernaski, and J. Camra, Synth. Met., 143, 341 (2004).

20. A. Mourato, A. S. Viana, J. P. Correia, H. Siegenthaler, and L. M. Abrantes, Electrochim. Acta, 49, 2249 (2004).

21. K.-M. Mangold, F. Meik, and K. Juttner, Synth. Met., 144, 221 (2004).

22. J. Tsuji, "Palladium Reagents and Catalysts: Innovations in Organic Synthesis," John Wiley, Chichester, 1995, p. 1.

23. C. Liao and M. Gu, Thin Solid Films, 408, 37 (2002).

24. Y. Chen, E. T. Kang, and K. G. Neoh, Appl. Surf. Sci., 185, 267 (2002).

25. S.-J. Choi and S.-M. Park, J. Electrochem. Soc., 149, E26 (2002).

26. M. Campos and B. Bello, Jr., Synth. Met., 60, 1 (1993).

27. A. Eftekhari, M. Kazemzad, and M. Keyanpour-Rad, Appl. Surf. Sci., 239, 311 (2005). 